Research Paper

\title{
Human Stromal Cells in the Peripheral Zone of the Prostate Promote Tumorigenesis of Prostatic Cancer Stem Cells through Up-regulation of C-Kit Expression
}

\author{
Yubing Peng1\#, Qi Chen"1\#, Meng Gu1\#, Yanbo Chen1, Ming Zhang11, Juan Zhou'1, Hao Wang1, Yan Gao², \\ Wenji Li, Zhong Wang ${ }^{\circledR}$ and Zhikang Cai ${ }^{\circledR}$ \\ 1. Department of Urology, Ninth People's Hospital Affiliated to Shanghai Jiaotong University School of Medicine, Shanghai, 200011, China; \\ 2. Department of Infectious Diseases, Huashan Hospital, Fudan University, Shanghai, 200040, China \\ \# These three authors contributed equally to this study.
}

Corresponding authors: Dr. Zhong Wang, 639 Zhi Zaoju Road, Shanghai, 200011, Tel: 86-13301980998, Email: zhongwang2001@yahoo.com; Mr. Zhikang Cai, 639 Zhi Zaoju Road, Shanghai, 200011, Tel: 86-13311630565, Email: 13311630565@189.cn

(c) 2015 Ivyspring International Publisher. Reproduction is permitted for personal, noncommercial use, provided that the article is in whole, unmodified, and properly cited. See http://ivyspring.com/terms for terms and conditions.

Received: 2014.06.22; Accepted: 2014.09.05; Published: 2015.07.03

\begin{abstract}
Objective: Most prostate cancers originate from the prostatic peripheral zone (PZ). We tested the hypothesis that the stromal cells from PZ and transitional zone (TZ) have differential effects on the ability of tumorigenesis.

Methods: Stromal cells isolated from the PZ and TZ of normal human prostates mixed with DU145 cells subcutaneously injected into athymic nude mice. The volume and weight of tumors was measured and analyzing the ability of purified DU1 45 cells isolated from the tumors to migrate and proliferate. The expression patterns of stem cell-specific genes of these DU145 cells were examined. The C-Kit inhibitor, imatinib mesylate, was administrated to confirm the effect of stromal cells on the tumorigenesis.

Results: The volume and weight of tumors were significantly higher in mice transplanted with DU145 and stromal cells from PZ. In contrast, the data was significantly lower with DU145 and stromal cells from TZ than DU145 alone. The purified DU145 cells isolated from the tumors with DU145 and stromal cells in PZ had increased ability to migrate and proliferate, and had increased expression of C-Kit. These effects of the stromal cells in the PZ on DU145 cells could be blocked using imatinib mesylate.

Conclusions: Human stromal cells in the PZ promote the in vivo tumorigenesis of DU145 through up-regulating C-Kit; in contrast, the stromal cells in the TZ inhibit it through down-regulating the expression of $\mathrm{C}$-Kit. The model will be useful for understanding the mechanisms by which the prostatic stem cell niche controls the tumorigeneis of prostatic cancer stem cells.
\end{abstract}

Key words: prostatic cancer stem cell; stromal cells; peripheral zone; transitional zone; C-Kit

\section{Introduction}

Prostate cancer, the most commonly diagnosed malignancy, has become the second leading cause of cancer-related deaths among men in the United States [1]. Prostate cancer often grows slowly. For many patients, when diagnosed, their prostate cancer has al- ready developed into metastatic cancer; thus, hormone ablation therapy is the only choice for those patients.

Most human prostate cancers are adenocarcinomas, which originate from the epithelial cells that 
line the glands and ducts of prostate [2]. These glands and ducts are distributed in the prostate and surrounded by stromal tissues. A prostate has four distinct glandular zones: the peripheral zone (PZ), central zone, transitional zone (TZ), and anterior fibro-muscular zone. $70-80 \%$ of prostate cancers originate from the PZ, and 20-30\% from the TZ. Most prostate cancers from the $\mathrm{TZ}$ exhibit relatively low malignancy. However, the underlying mechanisms for the differential cancer origination and malignancy remains unknown [3]. The study to elucidate these mechanisms has been hindered due to the lack of relevant animal models.

Hormone therapy for most patients with prostate cancer aims to remove or block the function of the male hormone androgen. However, many patients eventually develop a condition in which cancer cells are refractory to the hormone therapy; this condition may be due to the existence of the cancer stem cells (CSCs) that are resistant to the hormone therapy.

CSCs have been isolated from many kinds of solid cancers [4]. Like normal adult stem cells, CSCs also have the ability to self-renew and to differentiate into many types of cells depending on their origin. The self-renewal and differentiation of CSCs is controlled by intrinsic and extrinsic factors. The intrinsic factors were suggested by the evidence that stem-cell-like populations are often found in immortalized cancer cell lines [5]. The extrinsic factors mainly exist in stem cell niches, which are microenvironments interacting with stem cells to regulate their fate. It has been thought that a niche provides stromal elements, which play an important role in regulating the differentiation of CSCs [6]. For example, an epithelial-mesenchymal transition (EMT) can induce human immortalized mammary epithelial cells into mesenchymal cells expressing CSC markers [7]. After detaching from other cells and the extracellular matrix, cells in the EMT migrate through the lymphatic or vascular system to other organs where they colonize [8]. Supporting this conclusion, Mani et al [7] found that EMT activators, such as Twist1 and SNAIL1, up-regulate the expression of the CSC markers in breast cancer. Furthermore, mammary epithelial cells undergoing EMT acquire the properties of mesenchymal stem cells (MSCs) from human bone marrow. Mammary epithelial cells have similar MSC surface markers, and possess multiple differentiation and increased migratory capacities [9]. Thus, any epithelial cancer cells may become CSCs when processed through EMT.

CSCs also exist in prostate cancer. In a prostate cancer mouse model, castration causes the rapid apoptosis of over $90 \%$ of prostate luminal cells [10]. Two weeks later, the regressed prostate epithelia be- came castration-resistant, implying the existence of hormone-independent stem cells. Prostatic CSCs were later isolated as a small population of cells expressing $\mathrm{CD}_{4} 4^{+} /$alpha2beta $1^{+} / \mathrm{CD} 133^{+}$surface markers [5].

CSCs have also been identified in some prostatic cancer cell lines with different responses to androgen. For example, both PC3 and DU145 cells proliferate in the absence of androgen, but only PC 3 cells are still sensitive to androgen. In PC3 cells, cells expressing CD44 and CD133 were found and showed stem cell properties [11]. In DU145 cells, cells expressing CD34 and C-KIT were isolated, but did not show stem cell properties in vitro [5].

The niche of prostatic CSCs may play an important role in regulating the self-renewal and differentiation of prostatic CSCs. Zhao et al. found that PC3 cells showed increased tumorigenic ability after being cultured with the stromal cells isolated from either the PZ or TZ of normal human prostates; this increased tumorigenic ability was more obvious after PC3 cells were cultured with stromal cells from the PZ than from the TZ [12]. Peng et al. found that DU145 cells also showed increased ability to proliferate and migrate after being cultured with stromal cells isolated from the PZ of normal human prostates [13]. These observations suggest that stromal-epithelial interactions may regulate the stem cell properties of CSCs and, thus, alter their tumorigenic ability.

We hypothesize that stromal cells from the PZ and TZ of normal human prostates may differ in their regulation of DU145 cells' ability to generate tumors and to maintain their stem cell properties. To test this hypothesis, we co-transplanted DU145 cells with stromal cells isolated from the PZ and TZ of normal human prostates into nude athymic mice to analyze the tumorigenesis and the stem-cell properties of the DU145 cells. The effects of an inhibitor of stem cellmarker, c-Kit, on the tumorigenesis and the stem-cell properties of DU145 cells were also analyzed.

\section{Materials and methods}

All human sample-related experiments were approved by the Ethics Committee of the School of Medicine, Shanghai Jiaotong University (Shanghai, China)

\subsection{Isolation and culture of primary prostate stromal cells}

The isolation and culture of primary prostate stromal cells was carried out as previously described [13]. To trace normal prostate-derived stromal cells, we labeled isolated prostate stromal cells with $10 \mu \mathrm{m}$ carboxyfluorescein succinimidyl ester (CFSE) (Invitrogen, CA) from a labeling kit according to the man- 
ufacturer's instructions.

Purification of DU145 cells from the tumors of an in vivo tumorigenesis mouse model was carried out as previously described [13].

Human primary prostate stromal cells were isolated from the prostates of three male donors aged 22,23 , and 40 years. Isolated cells were cultured in RPMI-1640 supplemented with 10\% FBS, 100U/mL penicillin, and $100 \mu \mathrm{g} / \mathrm{mL}$ streptomycin at $37^{\circ} \mathrm{C}$ with $5 \% \mathrm{CO}_{2}$. The growth medium was changed every 2 days. Cells with less than four passages were used in this study.

\subsection{In vivo tumorigenesis and administration of C-Kit inhibitor}

Male athymic nude mice at 4 weeks of age were purchased from the Experimental Animal Center of the Chinese Academy of Sciences (Shanghai, China). Mice were randomly divided into 6 groups with 8 to 10 mice in each group. Mice in each group were subcutaneously injected with different combination of cells as summarized in the Table 1. To administrate the C-Kit inhibitor imatinib mesylate into mice transplanted with various combinations of cells, these mice were intraperitoneally injected with the inhibitor at $0.05 \mathrm{mg}$ per gram of body weight per day from the day when cell injection was carried out. After 48 days, tumors from each mouse were harvested for isolation of epithelial cells.

Table 1. Summary of experimental design

\begin{tabular}{llllll}
\hline Group Name & $\begin{array}{l}\text { Number C-Kit } \\
\text { of Mice }\end{array}$ & $\begin{array}{l}\text { Inhibitor } \\
\text { Mice with } \\
\text { Tumor }\end{array}$ & $\begin{array}{l}\text { Number of } \\
\text { Mice that } \\
\text { Survived }\end{array}$ & $\begin{array}{l}\text { Survival } \\
\text { rates }\end{array}$ \\
\hline DU145 alone & 10 & No & 5 & 8 & $80 \%$ \\
DU145+PPSC & 10 & No & 8 & 8 & $80 \%$ \\
DU145+PTSC & 10 & No & 5 & 9 & $90 \%$ \\
DU145 alone & 8 & Yes & 5 & 6 & $75 \%$ \\
DU145+PPSC & 8 & Yes & 6 & 7 & $87.5 \%$ \\
DU145+PTSC & 8 & Yes & 6 & 6 & $75 \%$ \\
\hline
\end{tabular}

Two million of each cell type designated in the table were subcutaneously transplanted into each mouse. PPSC, peripheral zone stromal cells; PTSC, transitional zone stromal cells.

\subsection{Isolation of epithelial cells from primary tumors in mice}

Epithelial cells from the tumors of mice receiving cell transplantation were isolated and cultured as described previously [13]. Cells at a passage number of 6 to 10 were used for further experiments.

\subsection{Immunocytochemistry and immunohistochemistry}

For immunocytochemistry, the epithelial cells purified from the tumors and naive DU145 were first grown on a glass coverslip for $48 \mathrm{~h}$, and then were fixed in $4 \%$ paraformaldehyde. Fixed cells were stained with CD34 (1:400, Santa Cruz Biotech, Santa Cruz, CA), CD44 (1:200, Epitomics, Burlingame, CA), CD133 (1:400, Santa Cruz Biotech., Santa Cruz, CA), and C-KIT(1:200, Epitomics, Burlingame, CA) primary antibodies (mouse anti-human) followed by 1:2000 secondary antibody (anti-mouse IgG, CST, CA).

For immunohistochemistry, tumors were fixed in $4 \%$ paraformaldehyde solution and then dehydrated, sealed in wax, and cut. Cut tissue sections were stained with the primary antibodies as described previously [14]

To determine the stromal cell types isolated from the peripheral zone (PZ) and transitional zone (TZ), the cells were stained with primary antibodies against vimentin (a marker for mesenchymal cells, especially fibroblasts) (Epitomics, Burlingame, CA), a-SMA (a marker for smooth muscle cells and myofibroblasts) (Abcam, Cambridge, UK), smoothlin (a marker for smooth muscle cells) (Santa Cruz Biotech, Santa Cruz, CA), and cytokeratin 18 (CK18) (a marker for peripheral cells) ( Abcam, Cambridge,UK) as described previously [14]. CK18 was expressed in all four cell types and used to monitor the efficiency of the isolation and purification procedure.

\subsection{MTT assays}

DU145 cells were seeded on 96-well plates $(2 \times$ $10^{3}$ cells/well). After 24, 48, 72, 96, 120, and 144 hours of culturing, 3-4,5-dimethylthiazol-2,5 diphenyl tetrabromide (MTT) ( Sigma, St. Louis, MO, USA) was added into each well to achieve a final concentration of $5 \mathrm{mg} / \mathrm{ml} \mathrm{MTT}$. Cells were incubated at $37^{\circ} \mathrm{C}$ for another 2 hours to allow formation of purple formazan. The absorbance of the medium in each well was measured at $570 \mathrm{~nm}$.

\subsection{Wound healing migration assay}

Epithelial cells isolated from tumors and naive DU145 cells were seeded on a 12 -well plate $\left(1 \times 10^{4}\right.$ cells/well), and grown for 48 hours to approximately $90 \%$ confluence. A denuded zone at the center of the cell monolayer in each well was made by scratching with a sterile micropipette tip. Loosed cells were removed by washing with PBS. The denuded zones were photographed by microscopy at 0,12 and 24 hours. To minimize the interference from cell proliferation, cell proliferation was inhibited with $0.5 \mathrm{M}$ mitomycin C (Sigma, St. Louis, MO, USA).

\subsection{Quantitative real-time PCR analysis}

The mRNA levels of CD34, CD44, CD133, and $\mathrm{C}$-KIT were measured using quantitative real-time PCR (q-PCR). The mRNA levels of $\beta$-actin was used as an internal loading control. The total RNA from cul- 
tured cells was extracted using Trizol reagent and from tumor tissues using the RNA Prep Pure Tissue Kit (TIANGEN Biotech, Beijing, China) according to the manufacturer's instructions. Total RNA $(1 \mu \mathrm{g})$ from each sample were reversely transcribed to cDNA at $42^{\circ} \mathrm{C}$ for 60 minutes followed by inactivation at $94^{\circ} \mathrm{C}$ for 5 minutes. The cDNA then served as the template for SYBR green real-time PCR. The following primer pairs were used for qPCR: CD34: forward, 5'-CAACACCTAGTACCCTTGGAAGT-3', reverse, 5'-ACTGTCGTTTCTGTGATGTTTGT-3'; CD44: forward, 5'-ACCATGAAAACCAATCCCAGG-3', reverse, 5'-CCCATCCCAGACGAAGACAG-3'; CD133: forward, 5'-AAGAGAATGCCAATGGGTCCA-3', reverse, 5' -CATCCACAGATGCTCCTAAGGC-3'; C-KIT: forward, 5'-GCCTTTTCCGTGATCCATT CA-3', reverse, 5'-GGCGACGAGATTAGGC TGTTA-3'; $\beta$-ACTIN: forward, 5'-AGCGAGCA TCCCCCAAAGTT-3', reverse, 5'-GGGCACGAA GGCTCATCATT- $3^{\prime}$. The qPCR was run at $95^{\circ} \mathrm{C}$ for 2 minutes followed by 40 cycles of $95^{\circ} \mathrm{C}$ at 15 seconds, $55^{\circ} \mathrm{C}$ for 20 seconds, and $72^{\circ} \mathrm{C}$ for 45 seconds. Relative gene expression was calculated from the $\mathrm{Ct}$ value using the $\Delta \Delta \mathrm{Ct}$ method.

\subsection{Western blot}

Western blot was used to analyze the protein levels of C-Kit. Total protein was prepared from the harvested tumors. Proteins ( $50 \mu \mathrm{g})$ were separated by SDS-PAGE (Bio-Rad) and then transferred onto nitrocellulose membranes (Life Science Research;
Bio-Rad). Membranes were incubated with primary mouse anti-human c-Kit monoclonal antibodies (1:200) purchased from Epitomics, Burlingame, CA. Anti- $\beta$-actin monoclonal antibody (Sigma-Aldrich) and then secondary antibody (1:2000).

\section{Results}

\subsection{Characteristics of stromal cells isolated from normal human prostates}

The PZ and TZ of the normal human prostate tissues were defined according to the zonal anatomy proposed by McNeal [15]. The stromal cells were isolated and analyzed at passage four by immunohistochemical staining. All cells were found to express vimentin, a marker for stromal cells (Fig. 1C), but not CK18, an epithelial cell-specific marker (Fig. 1D), which suggests that the isolated cells were pure stromal cells.

The isolated stromal cells were further stained with anti-vimentin, a-SMA, and smoothelin to classify the cell types from the cells isolated from the PZ or TZ. All stromal cells from either the PZ or TZ were found to express the myofibroblast marker vimentin, while few cells were found to express a-SMA (7.67 $\pm 4.6 \%$ vs. $6 \pm 4 \%$ for PZ and TZ, respectively) and smoothelin $(2 \pm 1.5 \%$ vs. $3 \pm 3.3 \%$ for PZ and TZ, respectively) (Fig. 1C). No statistical significance was found between each cell type among the stromal cells isolated from PZ and TZ ( $>0.1)$.
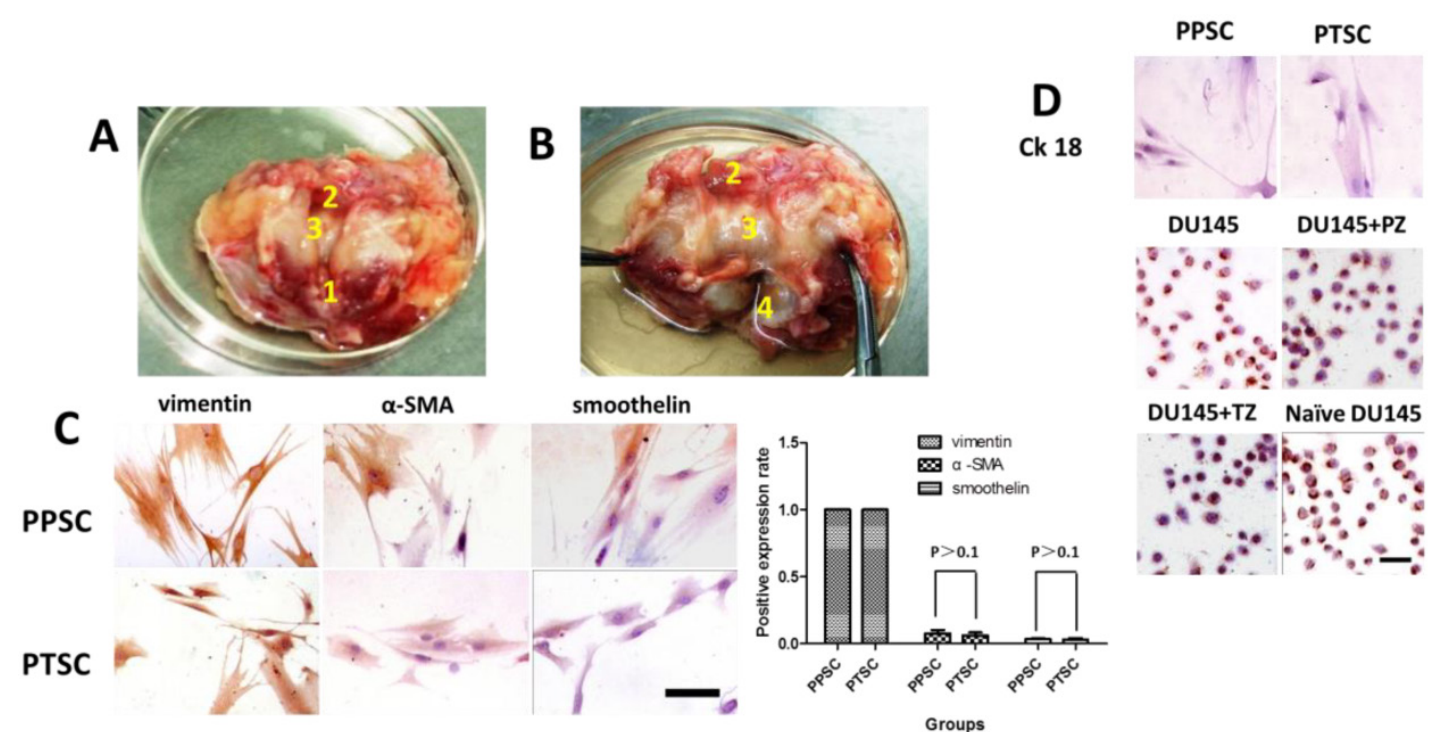

Figure 1. Anatomical structure of normal human prostate ( $A$ and $B$ ) and the characteristics of stromal cells isolated from the peripheral zone and the transitional zone of the normal human prostate (C). Isolated epithelial cells stained by anti-CK18 (D). A) A normal human prostate with the internal urethral orifice up. B) A normal human prostate with an incision made along the urinary tract. The posterior part of prostate gland is on the bottom. 1 , verumontanum; 2 , the cystic triangle area; 3, urothelium covering the transitional zone (TZ); and 4, peripheral zone (PZ) of prostate. C) CK18 staining of DU145 cells (A, scale bar=100 $\mu$ m), Note: all isolated epithelial cells were positive for CK18, an epithelial cell-specific marker. D) Stromal cells isolated from the PZ (PPSCs) and the TZ (PTSCs) of human prostates were grown in 6 -well plate sfor four passages and stained with antibodies against vimentin (left), $\alpha$-SMA (middle), and smoothelin (right). Scale bar=100 $\mu$ m. All the PSCs exhibited $100 \%$ positive anti-vimentin staining. The $\alpha$-SMA and smoothelin positive expression rates between PPSCs and PTSCs were not statistically different. 

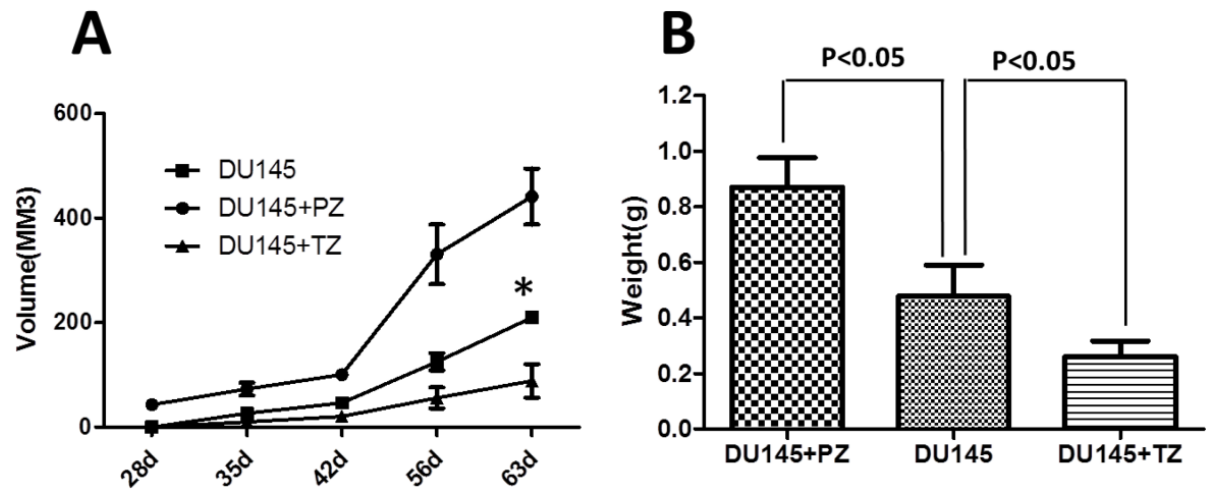

Figure 2. In vivo tumorigenesis of DU145 cells mixed with the stromal cells from the PZ or TZ of normal human prostates. DU145 cells mixed with or without the stromal cells from the PZ or the TZ were subcutaneously injected into athymic nude mice. The volume of tumor (A) in each mouse was measured every week from four weeks to nine weeks after cell injection. The weight of tumor (B) in each mouse was measured after a tumor was harvested at the end of experiment. Numbers are presented as the average \pm standard derivation of 10 samples from each group. DU145, group of mice transplanted with DU145 cells; DU145+PZ, group of mice transplanted with DU145 mixed with the stromal cells from the PZ; DU145+TZ, group of mice transplanted with DU145 mixed with the stromal cells from the TZ. *P $<0.05$ vs. DU145 group. \#P $<0.05$ vs. DU145+TZ group.

\subsection{Effects of human stromal cells from the PZ and TZ on the tumorigenesis of DU145 cells in mice}

To evaluate the effects of stromal cells the from PZ and TZ on the in vivo tumorigenesis of DU145 cells, DU145 cells were subcutaneously injected with or without the stromal cells from the different zones into athymic nude mice and the tumors' growth monitored up to nine weeks after cell transplantation. In the DU145 group (nine mice), one mouse died and six mice formed tumors. In the DU145+PZ group (nine mice), one mouse died and eight mice formed tumors. In the DU145+TZ group (nine mice), all mice survived and five mice formed tumors. The average tumor volume (calculated as (length $\times$ width $\left.^{2}\right)$ / 2) in the DU145+PZ group was significantly larger than that in the DU145 and DU145+TZ groups from week 4 after cell transplantation (Fig. 2A). Interestingly, the average tumor volume in the DU145+TZ group was significantly smaller than that in the DU145 and DU145+PZ groups at weeks 8 and 9 after cell injection (Fig. 2A). Likewise, the average tumor weight in these groups on the last day of the experiment had the same pattern as the average tumor volume (Fig. 2B).

It is possible that the different tumor size in the different groups was caused by different proliferation rates or apoptotic rates of the stromal cells from the PZ and TZ. Therefore, we used CFSE labeling technology to trace injected stromal cells and found that the stromal cells from both the PZ and TZ persistently existed in tumors with similar fluorescence intensity (Fig. 3).

Taken together, these results suggest that the stromal cells from the PZ increased the tumorigenesis of the DU145 cells, whereas the stromal cells from the TZ decreased the tumorigenesis of the DU145 cells.

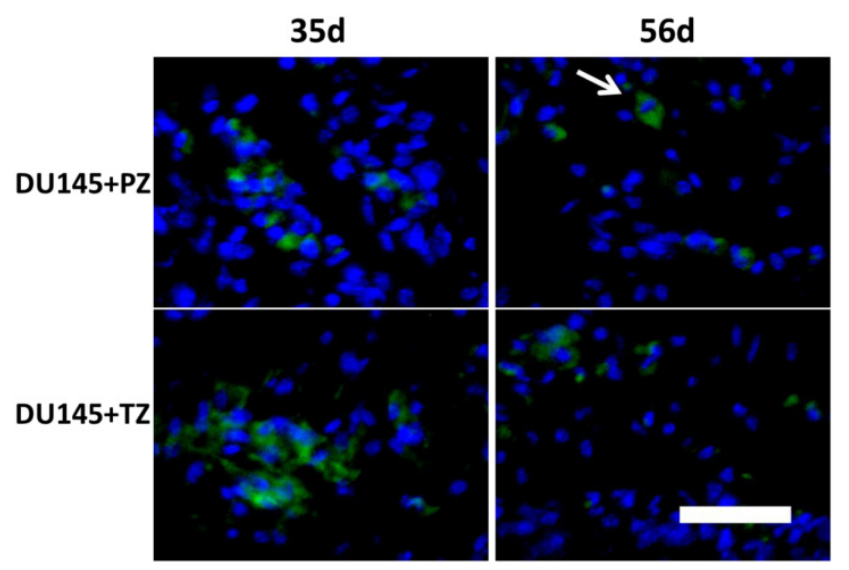

Figure 3. Stromal cells from PZ or TZ of human prostates had similar proliferation rate in mice. CFSE-labeled stromal cells mixed with DU145 cells were subcutaneously injected into nude athymic mice. Five or eight weeks later, tumors were harvested and sectioned; the fluorescence intensity in stromal cells was examined by fluorescence microscopy. No apparent difference in the fluorescence intensity of stromal cells from the PZ and the TZ was observed. Arrows point to CFSE-labeled fibroblast-like stromal cells. Scale bar $=100 \mu \mathrm{m}$

\subsection{Stromal cells from the PZ of normal human prostate increased the ability of DU145 cells to migrate and proliferate}

We then asked whether the stromal cells from the PZ or TZ altered the ability of DU145 cells to migrate and proliferate. At week nine after cell injection, the epithelial cells were purified from the tumors generated by subcutaneously injecting various combinations of cells as described above. Like the naive DU145 cells, purified epithelial cells from all three groups expressed CK18, an epithelial cell-specific marker (Fig.1D).

We tested the migration abilities of the DU145 cells isolated from the tumors in three groups of mice as described above and found that the DU145 cells from the DU145+PZ group migrated significantly more quickly than those from the other two groups or 
naive DU145 cells. No apparent difference in migration ability was observed among the naive DU145 cells and the DU145 cells from the DU145 and DU145+TZ groups (Fig. 4B).

Using the MTT assay, we found that the DU145 cells isolated from tumors in the DU145+PZ group proliferated significantly more quickly than those from the other groups (Fig. 4C).

\subsection{Altered expression of cancer stem-cell specific markers in the tumors formed in mice transplanted with various combinations of cells}

We then investigated the expression profiles of stem cell markers in the tumors formed in mice transplanted with the various combinations of cells.
The expression of CD34, CD44, CD133, and C-Kit were analyzed by immunohistochemical staining, qPCR, and Western blot. The expression of CD133 and C-Kit was significantly higher in the tumors formed in mice transplanted with DU145 cells mixed with the stromal cells from PZ (DU145+PZ) than in the other groups (Fig. 5).

The expression patterns of these stem cell markers were also analyzed in the epithelial cells purified from the tumors in these groups of mice using the same methods described above. We found that the expression of C-Kit was significantly higher in the cells from the DU145+PZ group than in those from the other groups, including naive DU145 cells (Fig. 6).

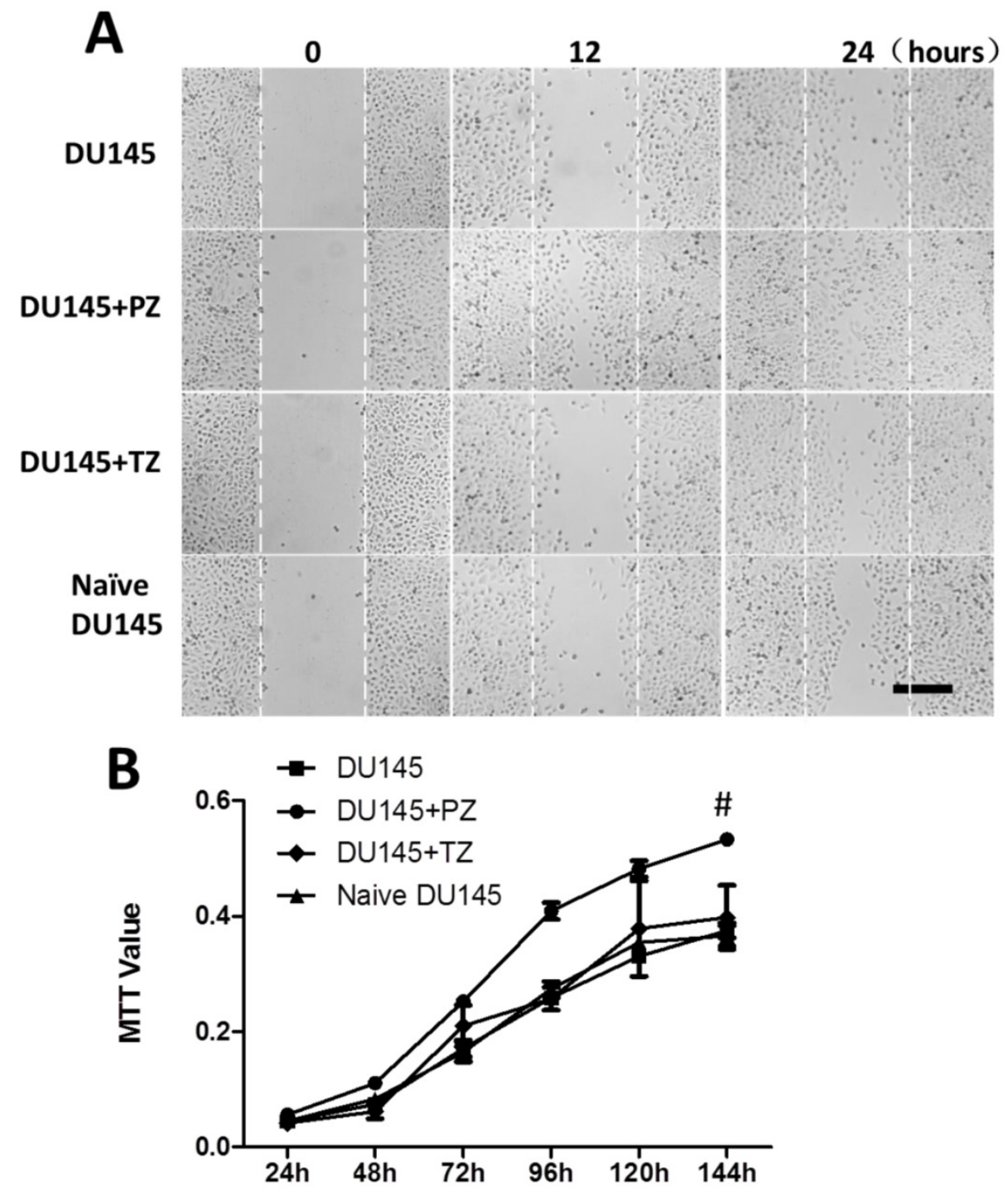

Figure 4. Effects of stromal cells from the PZ or TZ of human prostates on the migration and proliferation of DUI 45 cells. In vivo tumorigenesis was carried out as described in the legend of Fig. 2 . The stromal cells from the PZ of human prostates increased the migration ability (A) and proliferation rate (B) of DU145 cells. \#P $<0.05$, DU145+PZ vs. either of the other three groups. 


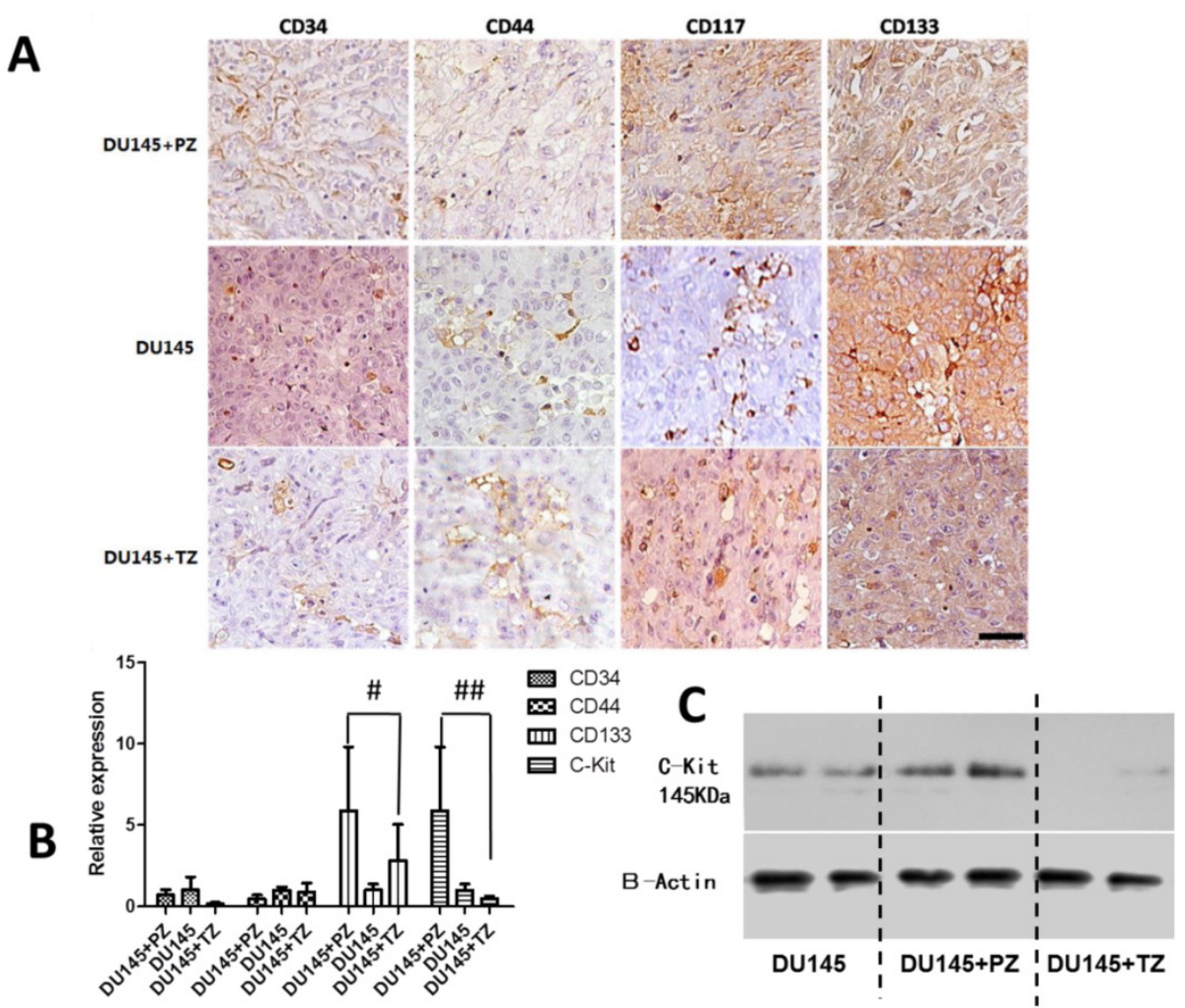

Figure 5. Analysis of expression of prostate cancer stem cell markers in tumors formed in mice transplanted with various combinations of cells. A) Immunohistochemical staining for prostate cancer stem cell markers, CD34, CD44, C-KIT, and CD 133 (scale bar $=100 \mu \mathrm{m}$ ). B) qPCR analysis for the mRNA levels of prostate cancer stem cell markers. The mRNA levels of each marker gene is presented as a ratio to the mRNA levels of $\beta$-actin. $\# P<0.01$ DU145+PZ vs. either of the other two groups; \#P<0.05 DU145+PZ vs. either of other two groups. C) Western-blot analysis for C-Kit showing that PZ stromal cells increased, but TZ stromal cells decreased the expression of C-Kit in DU145 compared to DU145 alone group.

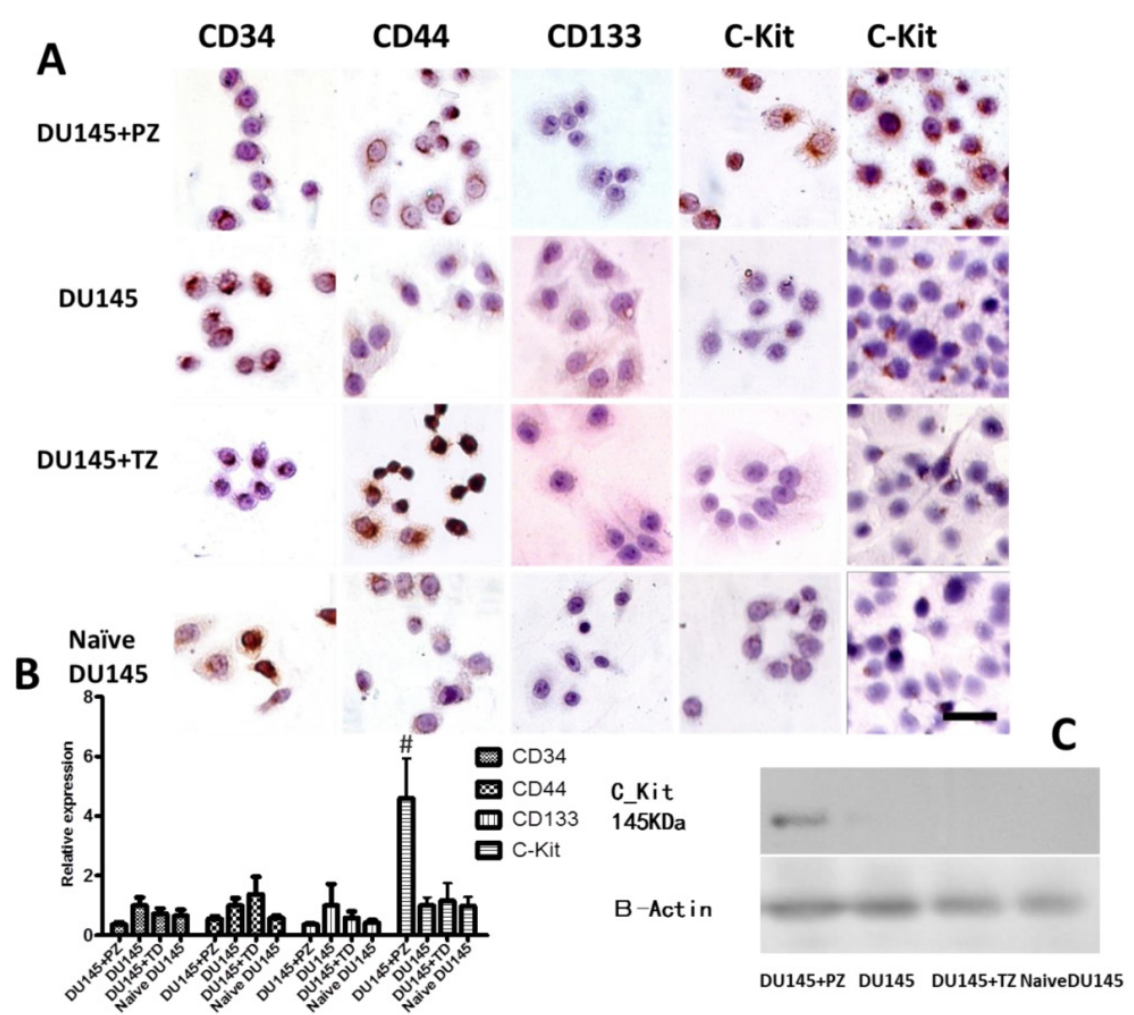

Figure 6. Expression of prostate cancer stem cell markers in the epithelial cells isolated from tumors formed in mice transplanted with various combinations of cells. A) Immunocytochemical assay for prostate cancer stem cell markers (scale bar $=100 \mu \mathrm{m}$ ). B) qPCR analysis for the mRNA levels of prostate cancer stem cell markers; the mRNA levels of each marker is presented as a ratio to the mRNA levels of $\beta$-actin. \#P<0.01 PD (DU145 co-cultured with peripheral stromal cells) vs. the other three groups (DU145 co-cultured with transitional stromal cells, DU145 alone and DU145 cells without cultured in vivo). C) Western blot analysis for C-Kit showing C-Kit expression was only detected in DU145+PZ group. 


\subsection{Effects of the C-Kit inhibitor imatinib mesylate on the tumorigenesis of DU145 cells mixed with the stromal cells from the PZ of normal human prostate}

Because the stromal cells from the PZ of normal human prostates significantly increased the C-Kit expression in the epithelial cells of tumors, we investigated the inhibitory role of a C-Kit inhibitor, imatinib mesylate, on the tumorigenesis in the mice transplanted with DU145 cells mixed with the PZ stromal cells. The administration of imatinib mesylate significantly reduced the average tumor size in the mice transplanted with DU145 cells and the PZ stromal cells from day 54 to 63 after cell injection, while this inhibition of tumorigenesis was not apparent in the mice transplanted with DU145 cells only (Fig. 7A and $B$ ).

Because we observed up-regulated C-Kit expression in the epithelial cells of tumors in the DU145+PZ group of mice, we thus asked whether the inhibition of tumorigenesis conveyed by imatinib mesylate decreased the expression of C-Kit in the epithelial cells using Western blotting. We found that, in both groups of mice (DU145 and DU145+PZ), administration of imatinib mesylate apparently decreased the C-Kit expression in the epithelial cells of tumors (Fig. 7C).

\section{Discussion}

In this study, we asked whether the stromal cells in the PZ and TZ of the normal human prostate could affect the in vivo tumorigenesis of DU145 cells, a commonly used prostatic cancer cell line. We found that the stromal cells in the PZ of normal human prostate promoted the in vivo tumorigenesis of DU145 cells through up-regulating the expression of C-Kit. This conclusion was based on our observation that the average size and weight of tumors formed in mice transplanted with the DU145 cells and the PZ stromal cells was significantly larger than those transplanted with the same number of DU145 cells alone (Fig. 2). In addition, we also observed increased migration and proliferation ability (Fig. 4B and C) and increased expression of CD133 and C-Kit (Fig. 5 and 6) in DU145 cells when these cells were co-injected with the PZ stromal cells. When a C-Kit inhibitor, imatinib mesylate, was given to the mice transplanted with DU145 cells and the PZ stromal cells, the tumorigenic ability of DU145 was dramatically reduced (Fig. 7).

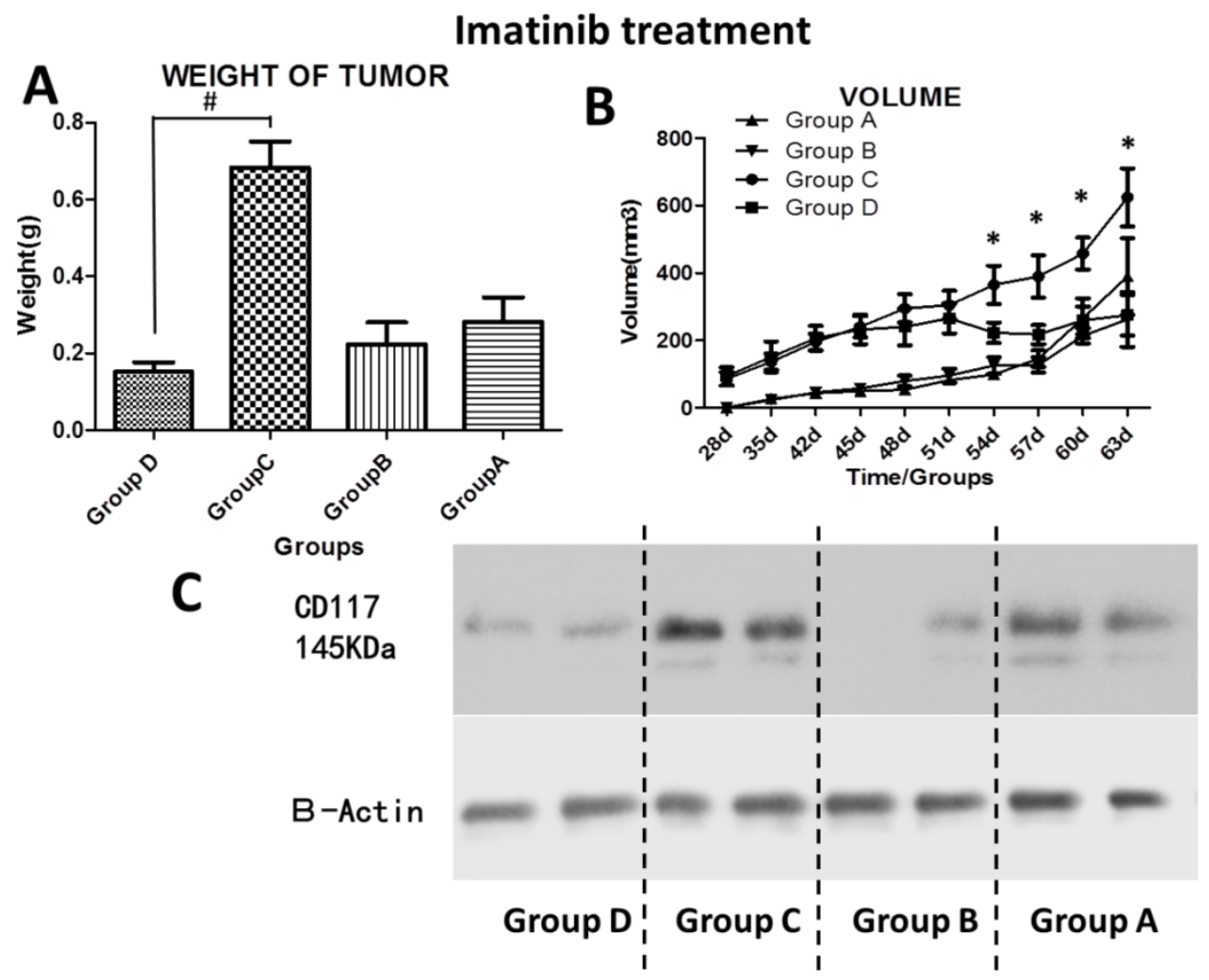

Figure 7. Effects of the c-Kit inhibitor imatinib mesylate on the in vivo tumorgenesis in mice transplanted with various combinations of cells. DU145 cells mixed with or without the stromal cells from the PZ were subcutaneously injected into athymic nude mice. Half of the mice in each group were intraperitoneally injected with imatinib mesylate. The volume of tumor $(A)$ in each mouse was measured every week from four weeks to nine weeks after cell injection. The weight of tumor (B) in each mouse was measured after a tumor was harvested at the end of experiment. Numbers are presented as the average \pm standard derivation of survivedsamples from each group. $* \mathrm{P}<0.05$, group C (DU145+PZ group) vs. either of the other three groups (group A: DU145, group B: DU145+ imatinib, group D: DU145+PZ+imatinib). \#P<0.05, group C vs. group D. C) Western blot for C-Kit of epithelial cells from tumors showed reduced expression of C-Kit in the tumors from the mice treated with imatinib mesylate. 
In contrast, we found that the stromal cells in the TZ inhibited the in vivo tumorigenesis of DU145 through down-regulating the expression of C-Kit. This conclusion was based on the observation that the volume and weight of tumors formed in mice transplanted with DU145 and TZ stromal cells was significantly decreased compared to those formed in mice transplanted with DU145 cells alone (Fig. 2). Furthermore, C-Kit expression in DU145+TZ was lower when compared with the tumors formed in mice transplanted with DU145 alone (Fig. 5 and 6). These opposite effects of PZ and TZ stromal cells on the in vivo tumorigenesis of DU145 cells might imply there are two different niches for prostatic CSCs: the PZ promotes the transition of the prostatic CSCs to metastasis and the TZ inhibits this transition process. This speculation was further supported by the opposite expression patterns of C-Kit and CD133 in tumors formed in these two groups of mice.

We previously investigated the effect of the stromal cells isolated from PZ or TZ on the tumorigenesis of $\mathrm{PC} 3$, another commonly used prostatic cancer cell line, and found that the stromal cells from both the PZ and TZ promote the in vivo tumorigenesis of PC3 cells in mice even though we observed that the PZ stromal cells had more profound effect than TZ stromal cells [13]. The different response of PC3 and DU145 cells to the stromal cells from the PZ and TZ may be caused by the different sensitivity of these cells to androgen. Although both PC3 cells and DU145 cells proliferate in the absence of androgen, only PC3 cells are sensitive to androgen. We also previously found that DU145 cells and stromal cells from the PZ did not form tumors in castrated nude mice. The androgen-insensitive DU145 cells may need androgen-mesenchymal interaction for tumorigenesis.

The niche of a type of CSCs provides a micro-environment to maintain the self-renewal of CSCs and to simultaneously drive some CSCs to proliferate, differentiate, and metastisize [16-17]. Therefore, the stromal microenvironment plays a critical role in tumor progression. In normal human prostates, stromal cells support and direct prostatic stem cells for epithelial differentiation and development via stromal-to-epithelial signaling [18]. In human cancers, including prostatic cancer, the tumor-associated stromal cells have been identified to have potential effects on the differentiation of CSCs [19-20]. However, these studies were mainly carried out in cell culture systems, which cannot represent the pathophysiologic features of the PZ and TZ stromal cells. Here, we used an in vivo tumorigenesis mouse model to study the effects of normal human PZ and TZ stromal cells on the tumor development of DU145 cells, an androgen-receptor-defective cancer cell line. We be- lieve that our in vivo tumorigenesis mouse model can more accurately represent the real niche for understanding the mechanisms by which prostatic CSCs are regulated.

The dramatic up-regulation of C-Kit in the DU145 cells of tumors formed in mice transplanted with DU145 and PZ stromal cells is quite intriguing. As one of the markers of the prostatic CSCs, the high expression of C-Kit is associated with high malignancy and bone metastases of prostatic cancer [21-22], and was found in the castration-resistant prostatic cancer mouse model [23]. In our in vivo tumorigenesis mouse model, the increased C-Kit expression was not only found in tumors with high malignancy, but was also maintained in the isolated and passaged epithelial cells, suggesting that our in vivo tumorigenesis mouse model may be a suitable model for understanding the mechanisms for prostatic cancer development.

Imatinib mesylate, a protein tyrosine kinase inhibitor, has been used to treat myelogenous leukemia and gastrointestinal stromal tumors by inhibiting their constitutively activated C-Kit pathway [24]. In this study, we showed that imatinib mesylate had a more considerable inhibitory effect on the tumorigenesis of DU145 cells in mice transplanted with DU145 and PZ stromal cells than those transplanted with DU145 cells alone (Fig. 7A and B). We also showed that imatinib mesylate decreased C-Kit expression, confirming that the up-regulation of C-Kit was at least one of the mechanisms by which PZ stromal cells affected the properties of prostatic CSCs. This result may be useful in developing strategies to treat prostatic cancer, especially, the hormone refractory prostatic cancer.

In conclusion, we found that PZ stromal cells promote the tumorigenesis of DU145 cells byup-regulating C-Kit expression; in contrast, TZ stromal cells inhibit this process. Furthermore, the established in vivo tumorigenesis mouse model may be useful for understanding the mechanisms by which the niche of prostatic CSCs regulate their properties.

\section{Acknowledgments}

This work was supported by grants from the National Natural Science Foundation of China (Grant Nos. 81172450, 81202008, and 81402089), the Projects of Shanghai Municipal Health Bureau (No. 20124Y160), and Key Disciplines Group Construction Project of Pudong Health Bureau of Shanghai (No. PWZxq2014-11).

\section{Competing Interests}

The authors have declared that no competing interest exists. 


\section{References}

1. Jemal A, Siegel R, Xu J, et al. Cancer statistics, 2010. CA Cancer J Clin. 2010; 60:277-300.

2. Long RM, Morrissey C, Fitzpatrick JM, Watson RW. Prostate epithelial cell differentiation and its relevance to the understanding of prostate cancer therapies. Clin Sci (Lond). 2005 Jan; 108(1):1-11.

3. Violette PD, Saad F. Chemoprevention of prostate cancer: myths and realities. J Am Board Fam Med. 2012 Jan; 25(1):111-9.

4. Erika Pastrana,Violeta Silva-Vargas,Fiona Doetsch. Eyes Wide Open: A Critical Review of Sphere-Formation as an Assay For Stem Cells. Cell Stem Cell. 2011 May 6; 8(5):486-98.

5. Chen W, Wang GM, Liu YJ. Cancer stem-like cells in human prostate carcinoma cells DU145: the seeds of the cell line? Cancer Biol Ther. 2007 May; 6(5):763-8.

6. Blum R, Gupta R, Burger PE. Molecular Signatures of the Primitive Prostate Stem Cell Niche Reveal Novel Mesenchymal-Epithelial Signaling pathways. PLoS One. 2010; 5(9): e13024.

7. Mani SA, Guo W, Liao MJ, Eaton EN, Ayyanan A, Zhou AY, Brooks M, Reinhard F, Zhang CC, Shipitsin M, Campbell LL, Polyak K, Brisken C, Yang J, Weinberg RA. The epithelial-mesenchymal transition generates cells with properties of stem cells. Cell. 2008 May 16; 133(4):704-15.

8. Pantel K, Brakenhoff RH. Dissecting the metastatic cascade. Nature Rev Cancer 2004; 4:448-456.

9. Battula VL, Evans KW, Hollier BG, Shi Y, Marini FC, Ayyanan A, et al. Epithelial-mesenchymal transition-derived cells exhibit multilineage differentiation potential similar to mesenchymal stem cells. Stem Cells 2010; 28:1435-45.

10. Tsujimura A, Koikawa Y, Salm S. Proximal location of mouse prostate epithelial stem cells: a model of prostatic homeostasis. J Cell Biol. 2002; 157(7):1257-65.

11. Fan X, Liu S, Su F, Pan Q, Lin T. Effective enrichment of prostate cancer stem cells from spheres in a suspension culture system. Urol Oncol. 2012; 30(3):314-8.

12. Zhao F-J, Han B-M, Yu S-Q, Xia S-J. Tumor formation of prostate cancer cells influenced by stromal cells from the transitional or peripheral zones of the normal prostate. AJA. 2009; :176-182.

13. Peng YB, Zhou J, Gao Y, et al. Normal prostate-derived stromal cells stimulate prostate cancer development. Cancer Sci. 2011; 102(9):1630-5.

14. Ramirez P, Del Razo LM, Gutierrez-Ruiz MC, Gonsebatt ME. Arsenite induces DNA-protein crosslinks and cytokeratin expression in the WRL-68 human hepatic cell line. Carcinogenesis. 2000; 21:701-6.

15. McNeal JE. The zonal anatomy of the prostate. Prostate . 1981; 2:35-49.

16. Iwasaki H, Suda T. Cancer stem cells and their niche. Cancer Sci. 2009; 100(7):1166-72.

17. Tomuleasa C, Kacsó G, Sorițău O, et al. Cellular interactions in prostate cancer genesis and dissemination. Looking beyond the obvious. Rom J Morphol Embryol. 2010; 51(3):427-35.

18. Joesting MS, Perrin S, Elenbaas B, et al. Identification of SFRP1 as a candidatemediator of stromal-to-epithelial signaling in prostate cancer. Cancer Res. 2005; 65(22):10423-30

19. Richardson AM, Woodson K, Wang Y, et al. Global expression analysis of prostate cancer-associated stroma and epithelia. Diagn Mol Pathol. 2007; 16 (4):189-97.

20. Pascal LE, Vêncio RZ, Goo YA, et al. Temporal expression profiling of the effects of secreted factors from prostate stromal cells on embryonal carcinoma stem cells. Prostate. 2009; 69(12):1353-65.

21. Simak R, Capodieci P, Cohen DW, Fair WR, Scher H, Melamed J, Drobnjak M, Heston WD, Stix U, Steiner G, Cordon-Cardo C. Expression of c-kit and kit-ligand in benign and malignant prostatic tissues. Histol Histopathol. 2000; 15(2):365-74.

22. Wiesner $\mathrm{C}$, Nabha SM, Dos Santos EB, Yamamoto $\mathrm{H}$, Meng $\mathrm{H}$, Melchior SW, Bittinger F, Thüroff JW, Vessella RL, Cher ML, Bonfil RD. C-kit and its ligand stem cell factor: potential contribution to prostate cancer bone metastasis. Neoplasia. 2008; 10(9):996-1003.

23. Leong KG, Wang BE, Johnson L, et al. Generationof a prostate from a single adult stem cell. Nature 2008; 456(7223): 804-8.

24. Bauer S, Corless CL, Heinrich MC, Dirsch O, Antoch G, Kanja J, Seeber S, Schütte J. Response to imatinib mesylate of a gastrointestinal stromal tumor with very low expression of KIT. Cancer Chemother Pharmacol. 2003 Mar;51(3):261-5 